Baltic Astronomy, vol.9, 183-195, 2000.

\title{
PRELIMINARY RESULTS FROM XCOV 17: PG 1336-018
}

M. D. Reed ${ }^{1}$, D. Kilkenny ${ }^{2}$, S. D. Kawaler ${ }^{1}$, A. Mukadam ${ }^{3}$, S. J. Kleinman ${ }^{1,4}$, A. Nitta-Kleinman ${ }^{3}$, J.L. Provencal ${ }^{5}$, T. Watson ${ }^{6}$, D. Sullivan ${ }^{7}$, T. Sullivan ${ }^{7}$, B. Shobbrook ${ }^{8}$, X. J. Jiang ${ }^{9}$, B. N. Ashoka ${ }^{10}$, S. Seetha ${ }^{10}$, E. Leibowitz ${ }^{11}$, P. Ibbetson ${ }^{11}$, H. Mendelson ${ }^{11}$, E. G. Meištas ${ }^{12}$, R. Kalytis ${ }^{13}$, D. Ališauskas ${ }^{13}$, D. O'Donoghue ${ }^{2}$, P. Martinez ${ }^{2}$, F. van $\mathrm{Wyk}^{2}$, R. Stobie ${ }^{2}$, F. Marang ${ }^{2}$, S. Zola ${ }^{14}$, J. Krzesinski ${ }^{14}$, W. Ogloza ${ }^{14}$, P. Mosaklik ${ }^{15}$, R. Silvotti ${ }^{16}$, A. Piccioni ${ }^{16}$ G. Vauclair ${ }^{17}$, N. Dolez ${ }^{17}$, J. Rene-Fremy ${ }^{17}$, M. Chevreton ${ }^{18}$, A. Ulla ${ }^{20}$, S. Dreizler ${ }^{19}$, S. Schuh ${ }^{19}$, J. Deetjen ${ }^{19}$, J.E. Solheim ${ }^{20}$, J. Perez ${ }^{20}$,

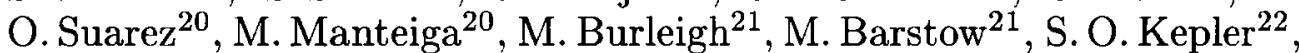
A. $\operatorname{Kanaan}^{22}$, O. Giovannini ${ }^{22}$, T. Metcalfe ${ }^{3}$ and R. Østensen ${ }^{20}$.

1 Department of Physics and Astronomy, Iowa State University, Ames, IA 50011, U.S.A.

2 South African Astronomical Observatory, PO Box 9, Observatory 7935, Cape Town, South Africa

3 Department of Astronomy, University of Texas, Austin, TX 78712, U.S.A.

4 Apache Point Observatory, P.O. Box 59, Sunspot, NM88349, U.S.A.

5 University of Delaware, Newark, DE 19716, U.S.A.

6 Southwestern University, 1001 E. University Avenue, Georgetown, Texas 78626, U.S.A.

7 School of Chemical and Physical Sciences, Victoria University of Wellington, P.O. Box 600, Wellington, New Zealand

8 Chatterton Astronomy Department, School of Physics, University of Sydney, NSW 2006, Australia

9 Astronomical Observatory, Academy of Sciences, Beijing 100080, China

10 Indian Space Research Organization, Airport Road, Vimanapura P.O., Bangalore 560017, India

11 Wise Observatory, Tel-Aviv University, Tel-Aviv 69978, Israel

12 Institute of Theoretical Physics and Astronomy, Goštauto 12, Vilnius 2600, Lithuania 
13 Institute of Material Research and Applied Sciences, Vilnius University, Ciurlionio 29, Vilnius 2009, Lithuania

14 Mt. Suhora Obsevatory, Cracow Pedagogical University, ul. Podchorażych 2, 30-084 Cracow, Poland

15 Copernicus Astronomical Center, ul. Bartycka 18, 00-716 Warsaw, Poland

16 Osservatorio Astronomico di Capadimonte, Via Moiariello 16, I-80131 Napoli, Italy

17 Université Paul Sabatier, Observatoire Midi-Pyrénées, CNRS/UMR5572, 14 av. E. Belin, 31400 Toulouse, France

18 Observatoire de Paris-Meudon, DAEC, 92195 Meudon, France

19 Institut für Astronomie und Astrophysik, Abteilung Astronomie, Waldhäuser Str. 64, D-72076 Tübingen, Germany

20 Institute of Mathematical and Physical Sciences, University of Tromso, P.O. Box 953, N-9001, Tromso, Norway

21 Astronomy Department, Leicester University, University Road, Leicester, LE17RH, UK

22 Instituto de Fisica UFRGS, Campus do Vale, C.P. 15051 Goncalves, Porto Alegre, RS, Brazil

Received December 7, 1999

\begin{abstract}
We present a preliminary analysis of the data collected during the 1999/1 (XCOV17) Whole Earth Telescope (WET) campaign to observe the pulsating subdwarf B star PG 1336-018. Since PG 1336-018 is an HW Vir type eclipsing binary, our hope is to use the known orbital period, assume a tidally locked system, and search for rotationally split modes. To this end, we can already report some success.
\end{abstract}

Key words: stars: subdwarf B, oscillations, individual: PG 1336018

\title{
1. INTRODUCTION
}

Pulsating horizontal branch stars with effective temperatures greater than $25000 \mathrm{~K}$ and $\log g$ greater than 5.0 make up the EC 14026 (or sdBV) class of stars. They are a subset of the subdwarf $B(\mathrm{sdB})$ population that is commonly found in sky surveys (PG survey: Green, Schmidt \& Liebert 1986, KPD survey: Downes 1986). These Extended horizontal branch (EHB) stars form a fairly homogeneous population of core He burning stars of approximately 
half a solar mass. They have been discovered in elliptical galaxies, globular and open clusters and in the field; though pulsators have only been confirmed in the field.

To date, there have been temporal spectra published for 13 EC 14026 stars (see O'Donoghue et al. 1999 for a review). However, these spectra tend to ask more questions then they answer. The periods range from 80 to 500 seconds, with some stars having as few as 2 modes while one has over 50 ! The periods lie in regions that encompass radial and non-radial modes, as well as $p$ and $g$ modes. With such a variety, it has been nearly impossible to make any mode identifications.

PG 1336-018 (hereafter PG 1336) was discovered to be a pulsating sdB star by Kilkenny et al. (1998), but with the added bonus that it is also an eclipsing binary. The companion is a late-type dwarf of type $\sim \mathrm{M} 5$, so the stellar radii are comparable yet the companion contributes little to the integrated flux. With an inclination of $\sim 81^{\circ}$ and a period of 2.4 hours, PG 1336 could provide some help in understanding the pulsation spectrum of EC 14026 stars. It is expected that PG 1336 is rotationally locked to it's companion, providing a known rotational velocity. Limited photometry by Kilkenny et al. (1998) provided two pulsation periods, with more indicated in the temporal spectrum.

This made PG 1336 an ideal candidate for a WET run. It was the co-primary target (along with BPM 37093) for the WET campaign XCOV 17, held in April 1999. In addition to the WET campaign, pre-WET data was obtained in March, providing a time-base of nearly two months. Here we present a preliminary reduction and analysis on the wealth of data obtained before and during XCOV 17.

\section{THE WEALTH OF DATA}

The idea behind the WET is to obtain continuous coverage of a target, with some redundancy for weather allowances. However, when there are two primary targets, some thin spots in coverage are revealed. Due to a lack of sites between McDonald Observatory (Texas) and B.A.O (China) a 3 hour gap appeared in every 24 hour cycle. This gap would normally have been covered by Siding Spring and Mount John, but these sites were dedicated to the southern primary target. So while XCOV 17 did not provide the usual WET coverage for PG 1336, it was still very successful as it provided excellent coverage for two targets. 
Table 1 lists the observatories involved in acquiring data for PG 1336 and the resulting data runs. Runs marked with an asterik were obtained outside of the official WET run by sites kind enough to begin early.

Table 1. Data acquired during XCOV 17.

\begin{tabular}{|c|c|c|c|c|}
\hline Telescope & Run name & $\begin{array}{l}\text { Date } \\
(\mathrm{UT})\end{array}$ & $\begin{array}{l}\text { Star time } \\
\text { (UT) }\end{array}$ & $\begin{array}{l}\text { (h:m:s) length } \\
\text { (h: }\end{array}$ \\
\hline Calar Alto $1.2 \mathrm{~m}$ & capg $1336 \mathrm{r} 1 * 1$ & 3 Apr 99 & $04: 17: 24$ & $\sim 45 \mathrm{~m}$ \\
\hline Calar Alto $1.2 \mathrm{~m}$ & capg1336r $2^{* 1}$ & 3 Apr 99 & $22: 06: 29$ & $\sim 6 \mathrm{~h} 48 \mathrm{~m}$ \\
\hline Calar Alto $1.2 \mathrm{~m}$ & capg $1336 \mathrm{r} 3^{* 1}$ & 4 Apr 99 & 21:00:04 & $\sim 8 \mathrm{~h} 06 \mathrm{~m}$ \\
\hline Wise $1 \mathrm{~m}$ & eml-001* & 5 Apr 99 & $19: 29: 11$ & $0: 34: 20$ \\
\hline Calar Alto $1.2 \mathrm{~m}$ & $\operatorname{capg} 1336 \mathrm{r} 4^{* 1}$ & 5 Apr 99 & $20: 40: 28$ & $\sim 8 \mathrm{~h} 20 \mathrm{~m}$ \\
\hline Calar Alto $1.2 \mathrm{~m}$ & $\operatorname{capg} 1336 \mathrm{r} 5^{* 1}$ & 6 Apr 99 & $20: 26: 06$ & $\sim 8 \mathrm{~h} 41 \mathrm{~m}$ \\
\hline Calar Alto $1.2 \mathrm{~m}$ & capg1336r $6^{1}$ & 7 Apr 99 & $21: 28: 00$ & $\sim 7 \mathrm{~h} 34 \mathrm{~m}$ \\
\hline Tenerife $0.8 \mathrm{~m}$ & iza-002 & 8 Apr 99 & $04: 37: 50$ & $1: 05: 45$ \\
\hline Calar Alto $1.2 \mathrm{~m}$ & $\operatorname{capg} 1336 \mathrm{r} 7^{1}$ & 8 Apr 99 & $21: 28: 00$ & $\sim 9 \mathrm{~h} 10 \mathrm{~m}$ \\
\hline Tenerife $0.8 \mathrm{~m}$ & iza-003 & 8 Apr 99 & $22: 54: 50$ & $5: 54: 20$ \\
\hline $\mathrm{BAO} 0.85 \mathrm{~m}$ & jxj-9902 & 9 Apr 99 & $13: 06: 40$ & $7: 17: 55$ \\
\hline Suhora $1 \mathrm{~m}$ & suh73 & 9 Apr 99 & $19: 42: 30$ & $7: 03: 15$ \\
\hline Calar Alto $1.2 \mathrm{~m}$ & $\operatorname{capg} 1336 \mathrm{r} 9^{1}$ & 9 Apr 99 & $20: 40: 00$ & $\sim 4 \mathrm{~h} 35 \mathrm{~m}$ \\
\hline Tenerife $0.8 \mathrm{~m}$ & iza-004 & 9 Apr 99 & $21: 46: 50$ & $8: 04: 45$ \\
\hline Calar Alto $1.2 \mathrm{~m}$ & $\operatorname{capg} 1336 \mathrm{r} 10^{1}$ & 10 Apr 99 & $00: 25: 45$ & $\sim 4 \mathrm{~h} 40 \mathrm{~m}$ \\
\hline Calar Alto $1.2 \mathrm{~m}$ & capg $1336 \mathrm{r} 11^{1}$ & 10 Apr 99 & $20: 52: 00$ & $\sim 2 \mathrm{~h} 22 \mathrm{~m}$ \\
\hline Loiano $1.5 \mathrm{~m}$ & lo1004 & 10 Apr 99 & $22: 30: 00$ & 4:58:05 \\
\hline Tene & iza- & 10 Apr 99 & $22: 41: 30$ & $6: 58: 00$ \\
\hline SAA & pm & 10 Apr 99 & $22: 42: 50$ & $0: 23: 20$ \\
\hline SAA & pm10 & 10 Apr 99 & $23: 30: 30$ & $1: 28: 20$ \\
\hline Cals & capg1336r & 11 Apr 99 & $00: 46: 00$ & $\sim 5 \mathrm{~h} 08 \mathrm{~m}$ \\
\hline $\mathrm{McL}$ & tkw-0 & 11 Apr 99 & $04: 30: 00$ & $5: 1$ \\
\hline Ten & iza-0 & 11 Apr 99 & $21: 52: 50$ & $5: 2$ \\
\hline SAA & $\mathrm{pm} 1$ & 11 Apr 99 & $22: 07: 20$ & $7: 3$ \\
\hline $2.1 \mathrm{~m}$ & tkw- & 12 Apr 99 & $06: 2$ & $1: 28: 20$ \\
\hline $\mathrm{BAC}$ & & 12 Apr 99 & $13: 49: 50$ & $6: 26: 00$ \\
\hline Suho & suh & 12 Apr 99 & $18: 55: 40$ & $1: 39: 15$ \\
\hline Wise & eml-00 & 12 Apr 99 & $18: 56: 40$ & $1: 44: 55$ \\
\hline SAA & $\mathrm{pmll}$ & 12 Apr 99 & $21: 00: 00$ & $6: 46: 25$ \\
\hline Tene & iza-00 & 12 Apr 99 & $22: 30: 50$ & $7: 02: 10$ \\
\hline $\mathrm{BA}$ & & 13 Apr 99 & $13: 10: 30$ & $6: 06: 35$ \\
\hline NainiTal $1 \mathrm{~m}$ & n49-0416 & 13 Apr 99 & $17: 12: 00$ & $5: 41: 45$ \\
\hline
\end{tabular}


Table 1. Continued.

\begin{tabular}{|c|c|c|c|c|}
\hline Telescope & Run name & $\begin{array}{l}\text { Date } \\
\text { (UT) }\end{array}$ & $\begin{array}{l}\text { Star time } \\
\left(\mathrm{U}^{\prime} \mathrm{T}\right)\end{array}$ & $\begin{array}{l}\text { Run length } \\
\text { (h:m:s) }\end{array}$ \\
\hline Tenerife $0.8 \mathrm{~m}$ & iza-008 & 13 Apr 99 & $21: 34: 20$ & $7: 55: 30$ \\
\hline Calar Alto $1.2 \mathrm{~m}$ & capg $1336 \mathrm{r} 14^{1}$ & 13 Apr 99 & $21: 32: 45$ & $\sim 1 \mathrm{~h} 21 \mathrm{~m}$ \\
\hline McDonald $2.1 \mathrm{~m}$ & tkw-0062 & 14 Apr 99 & 08:22:00 & $0: 35: 35$ \\
\hline NainiTal $1 \mathrm{~m}$ & $\mathrm{n} 49-0417$ & 14 Apr 99 & $17: 17300$ & $5: 48: 10$ \\
\hline Suhora $1 \mathrm{~m}$ & suh76 & 14 Apr 99 & $19: 18: 10$ & $6: 59: 35$ \\
\hline $\mathrm{SAAO} 1.9 \mathrm{~m}$ & fvw 187 & 14 Apr 99 & $22: 13: 36$ & $1: 54: 10$ \\
\hline SAAO $1.9 \mathrm{~m}$ & fvw 188 & 15 Apr 99 & $01: 24: 50$ & $1: 50: 50$ \\
\hline BAO $0.85 \mathrm{~m}$ & jxj-9905 & 15 Apr 99 & $12: 5$ & 7:01:00 \\
\hline Suhor & suh77 & 15 Apr 99 & 19:10:50 & 1:48:00 \\
\hline NainiTal $1 \mathrm{~m}$ & n49-0418 & 15 Apr 99 & $19: 38: 50$ & $1: 39: 25$ \\
\hline SAAO $1.9 \mathrm{~m}$ & fvw190 & 15 Apr 99 & $22: 27: 30$ & $3: 26: 40$ \\
\hline Suhora $1 \mathrm{~m}$ & suh78 & 15 Apr 99 & $22: 47: 50$ & $3: 52: 10$ \\
\hline McDonald $2.1 \mathrm{~m}$ & tkw-0064 & 16 Apr 99 & $03: 41: 30$ & $5: 54: 00$ \\
\hline Loiano $1.5 \mathrm{~m}$ & lo1604 & 16 Apr 99 & $19: 57: 52$ & $5: 19: 45$ \\
\hline McDonald $2.1 \mathrm{~m}$ & jlp-0160 & 17 Apr 99 & 04:36:00 & $6: 25: 45$ \\
\hline SAAO $1.9 \mathrm{~m}$ & fvw192 & 15 Apr 99 & 21:34:30 & $7: 16: 40$ \\
\hline McDonald $2.1 \mathrm{~m}$ & jlp-0162 & 18 Apr 99 & $03: 58: 20$ & $7: 17: 30$ \\
\hline $\mathrm{BAO} 0.85 \mathrm{~m}$ & jxj-9907 & 18 Apr 99 & $15: 17: 30$ & $3: 21: 00$ \\
\hline McDonald $2.1 \mathrm{~m}$ & jlp-0164 & 19 Apr 99 & 05:45:00 & $1: 38: 23$ \\
\hline McDonald $2.1 \mathrm{~m}$ & jlp-0165 & 19 Apr 99 & $07: 29: 15$ & $3: 33: 10$ \\
\hline Mt. John $1 \mathrm{~m}$ & ap1999q2 & 19 Apr 99 & $10: 38: 50$ & $0: 57: 10$ \\
\hline Mt. John $1 \mathrm{~m}$ & ap1999q3 & 19 Apr 99 & $11: 36: 30$ & $2: 35: 50$ \\
\hline NainiTal $1 \mathrm{~m}$ & $\mathrm{n} 49-0420$ & 19 Apr 99 & $17: 21: 30$ & $3: 52: 50$ \\
\hline Molètai $1.65 \mathrm{~m}$ & edroda04 & 19 Apr 99 & $20: 41: 40$ & $4: 23: 35$ \\
\hline McDonald $2.1 \mathrm{~m}$ & jlp-0167 & 21 Apr 99 & $03: 28: 20$ & $4: 32: 00$ \\
\hline McDonald $2.1 \mathrm{~m}$ & jlp-0168 & 21 Apr 99 & 08:10:29 & 2:54:00 \\
\hline NainiTal $1 \mathrm{~m}$ & $\mathrm{n} 49-0422$ & 20 Apr 99 & $16: 47: 10$ & $4: 37: 40$ \\
\hline
\end{tabular}

Note: ${ }^{1}$ CCD data.

During the 14 day period of XCOV 17, 172 hours of coverage were obtained, representing a duty cycle of $\approx 47 \%$. Including the sites that began early, we obtained 206 hours of coverage, or an overall duty cycle of $\approx 43 \%$. Though many hours of data were collected during the WET run, the longest continuous coverage was only 14 hours. We were also fortunate enough to collect over 30 hours of observations the month prior to the WET run and a single run about two weeks after XCOV 17. Table 2 lists the data obtained in March and May 1999. 
Table 2. Pre and post-WET data.

\begin{tabular}{llccc}
\hline Telescope & Run name & $\begin{array}{c}\text { Date } \\
\text { (UT) }\end{array}$ & $\begin{array}{c}\text { Star time Run length } \\
\text { (UT) }\end{array}$ & (h:m:s) \\
\hline McDonald 2.1 m & mdr019 & 8 Mar 99 & $10: 30: 00$ & $1: 35: 30$ \\
McDonald 2.1 m & mdr028 & 14 Mar 99 & $06: 48: 00$ & $4: 41: 15$ \\
McDonald 0.9 m & mdr031 & 17 Mar 99 & $04: 48: 00$ & $4: 35: 25$ \\
McDonald 0.9 m & mdr032 & 18 Mar 99 $09: 30: 00$ & $2: 31: 30$ \\
McDonald 0.9 m & mdr034 & 19 Mar 99 & $04: 34: 00$ & $7: 29: 00$ \\
McDonald 0.9 m & mdr036 & 20 Mar 99 $08: 29: 00$ & $3: 34: 30$ \\
Mt. John 1 m & ma2099q2 & 20 Mar 99 & $13: 42: 00$ & $3: 46: 45$ \\
McDonald 0.9 m & mdr038 & 22 Mar 99 & $03: 53: 30$ & $7: 57: 05$ \\
McDonald 0.9 m & mdr040 & 23 Mar 99 & $08: 08: 00$ & $1: 17: 55$ \\
Nordic 2.6 m & notpg1336r1 & 2 May 99 & $22: 27: 32$ & $6: 33: 10$ \\
\hline
\end{tabular}

Data acquisition and reduction of photomultiplier data followed the procedures outlined by Nather et al. (1990) with the following modification. Due to a strong reflection effect, and an eclipse every 1.2 hours, the program DFTWINPBAT was modified by Darragh O'Donoghue to extract eclipses based on the ephemeris of Kilkenny et al. (1998) and fit two sinusoids to remove the reflection effect. For the CCD data, it was slightly more complex. At this point, there is no standard WET CCD pipeline, so data arrive in various formats, and with non-standard timings.

Many telescopes place emphasis on deep imaging techniques, for which timing has little significance. As such, the format of the timestamp on CCD images varies considerably from site to site. For XCOV 17, CCD data for PG 1336 was obtained at Calar Alto, Spain and the Nordic Telescope at La Palma; each with a different timing method.

The data from the Nordic Telescope were provided by Roy $\emptyset$ stensen in a format adaptable to QED, so further reductions proceeded along the standard WET pipeline outlined by Nather et al. (1990). The data were received as raw counts from aperture photometry, with several choices for sky measurements available, including an annulus around the target star itself. The integrations were queued up, so they proceeded in equal time intervals, from start to finish. The cycle time (including integration) was 5.001 seconds but the time information stored in the image header was based on integration start time. In this case, it was a simple matter to convert the integration start time to the integration mid-point to fit the standard format. 

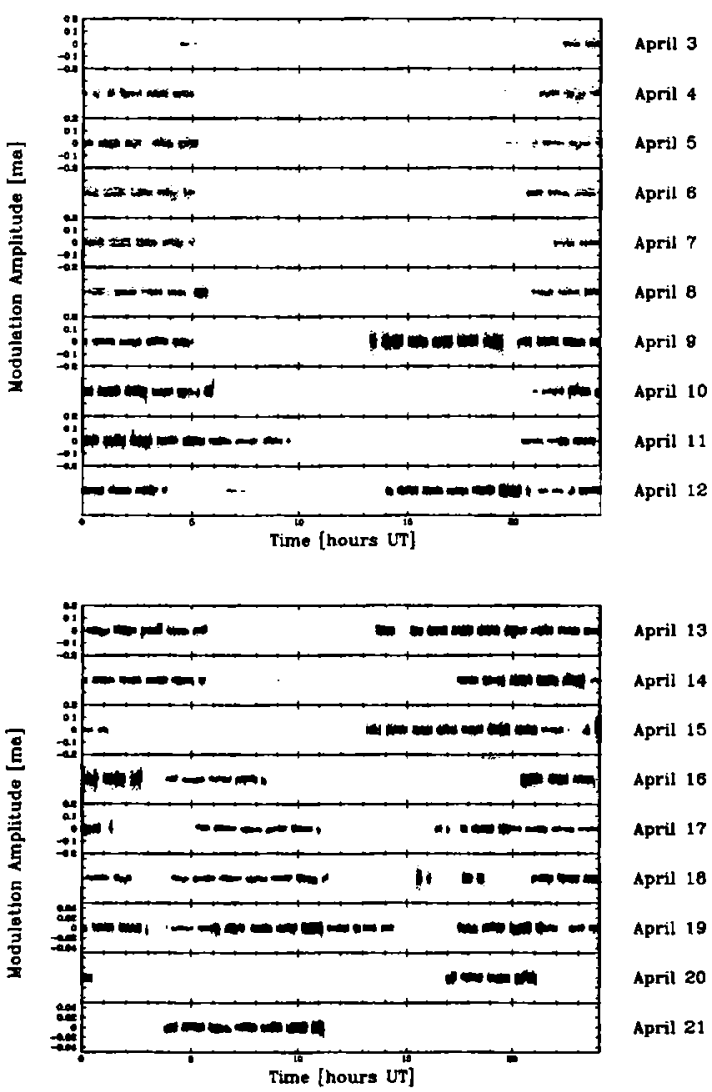

Fig. 1. Light curves for XCOV 17 data.

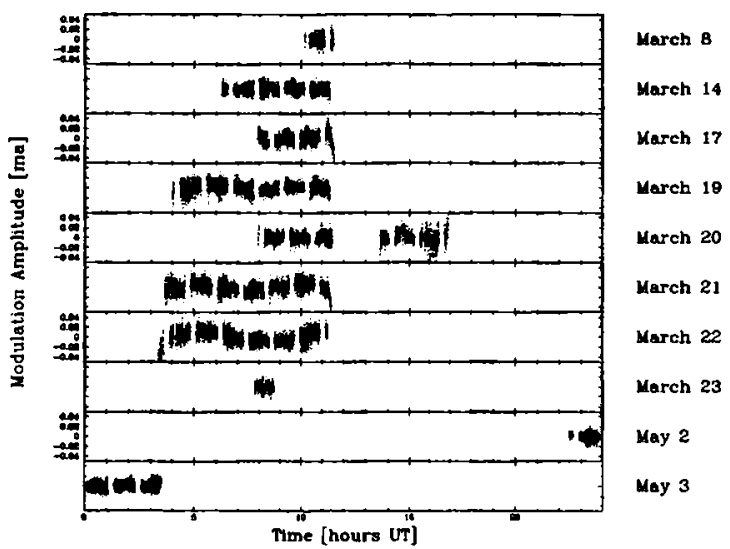

Fig. 2. Light curves for pre- and post-WET data. 


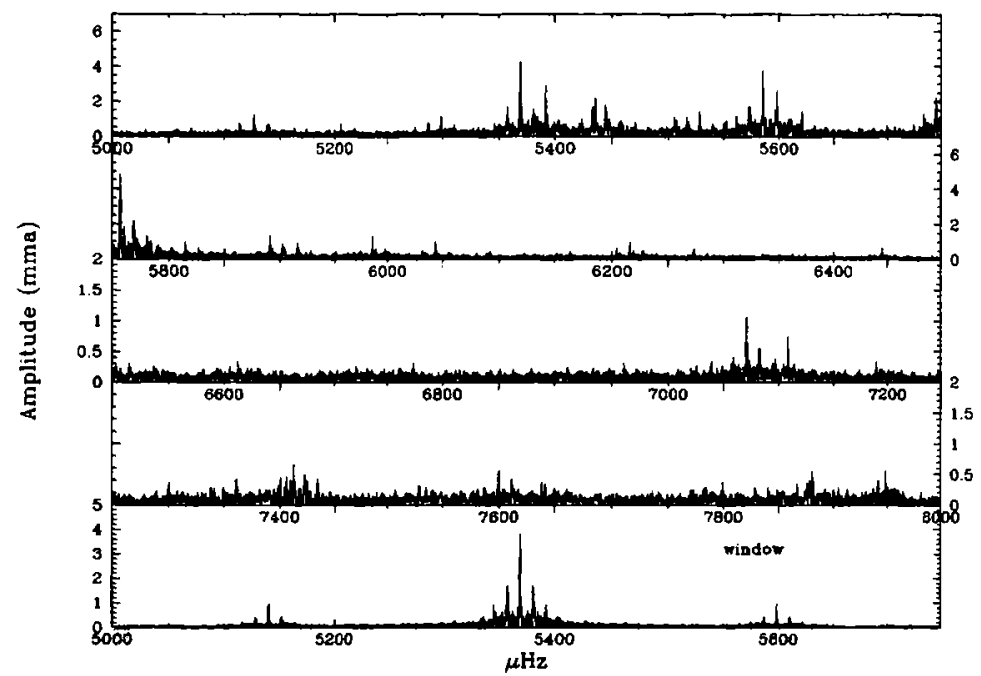

Fig. 3. The amplitude spectrum for the combined data set. Bottom panel is the spectral window. (Note: $y$ axis scale varies.)

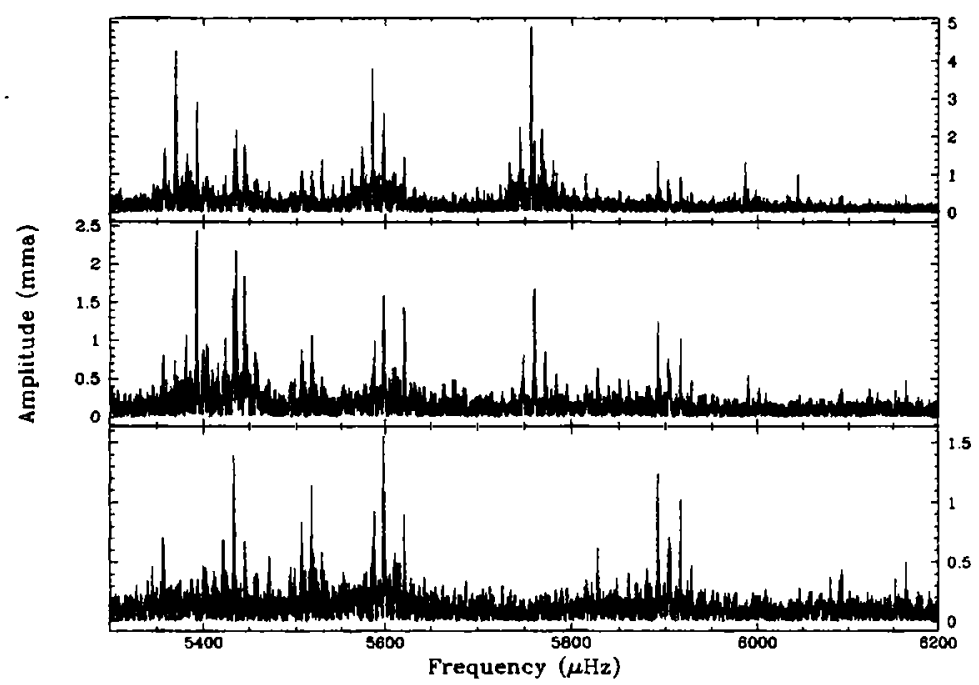

Fig. 4. Prewhitened frequency spectrum. The original frequency spectrum is at top, followed by spectra prewhitened by 3 and 7 periods. 
The format of the data acquired at Calar Alto was in three columns: time of integration beginning, in fractional Julian Day; relative flux of the target star (PG 1336) to a comparison star; and the error in the relative flux. A conversion program was written (by SDK) to convert fractional JD into first integration time, with the timing for subsequent integrations being seconds from the first integration. This program also accounted for leap seconds and provided barycentric corrections. However, the timing of these otherwise excellent runs had some problems. Again, the image header stored the integration start time, which had to be converted to integration midpoint, but the timing provided in the image header was less secure. The data acquisition computer did not directly read the GPS clock, and drifts of several seconds from GPS time were recorded during individual runs. Additionally, it still remains uncertain if the image header time was the actual time of the shutter opening or the time at which the computers began the process of integration which resulted in the opening of the shutter: a possible gap is of several tenths of a second.

Since the Calar Alto data were received as differential photometry rather than raw counts, some elements of the standard data reduction pipeline were unnecessary while others were more complicated. As both the target and comparison star were in the same field (and thus image), differential photometry accounted for sky extinction and variation. However, since extinction is wavelengthdependent, color differences between the stars manifested themselves as non-linear trends in the data. Bad points in the data were removed by hand, but the modified DFTWINPBAT expected sky/extinction corrected counts, rather than differential counts, so the data were multiplied by a constant (1000) and then later renormalized by the reduction software. Trends in the data that were not removed by the standard software were removed by the program POLYFILT, which fit a polynomial (second to fourth order in this case) to the data, and subsequently divided by it to remove the trends. Light curves for XCOV 17 data are shown in Fig. 1 while the light curves for the pre- and post- XCOV 17 runs are shown in Fig. 2.

\section{The pulsation spectrum}

The fourier transform of the complete data set is provided in Fig. 3 along with the data window. As the window shows, the temporal spectrum is complicated by daily aliases $(11.6 \mu \mathrm{Hz}$ from each 
peak) and by aliases from the eclipse extraction $(114.58 \mu \mathrm{Hz}$ from each peak). Twelve periods in the temporal spectrum were fit using linear least squares techniques with four additional periods found by visual inspection of the temporal spectrum. Table 3 provides a complete list of periods and amplitudes and Table 4 compares some of the frequencies detected to known aliases. Ten of the largest periods were then pre-whitened ( 7 of which are shown in Fig. 4). Though there appears to be remaining power in the spectrum (particularly after prewhitening), we cannot claim, with any confidence, that the remaining power is real.

Table 3. Period List for PG 1336 (with errors in parentheses).

\begin{tabular}{|c|c|c|c|c|c|c|c|}
\hline No. & Period(s) & & Frequency & $(\mu \mathrm{Hz})$ & Amplitud & $\mathrm{e}(\mathrm{mma})$ & Notes \\
\hline 1. & 186.24146 & $(7)$ & 5369.374 & $(2)$ & 3.655 & (7) & 1,2 \\
\hline 2. & 185.45663 & (14) & 5392.096 & (4) & 2.437 & (7) & 2 \\
\hline 3. & 184.05275 & (20) & 5433.225 & (8) & 1.315 & (6) & 3,4 \\
\hline 4. & 183.97798 & (17) & 5435.433 & (5) & 2.093 & (7) & 2 \\
\hline 5. & 183.67796 & (19) & 5444.311 & $(6)$ & 2.076 & (7) & 3,4 \\
\hline 6. & 181.63 & (2) & 5505.5 & $(6)$ & 0.842 & (15) & 5 \\
\hline 7. & 179.03049 & $(9)$ & 5585.641 & (3) & 4.085 & (7) & 1,2 \\
\hline 8. & 173.6923 & (13) & 5757.302 & (2) & 4.790 & (7) & 2 \\
\hline 9. & 173.58926 & (18) & 5760.725 & (6) & 1.643 & $(7)$ & 2 \\
\hline 10. & 169.7361 & (57) & 5891.5 & (2) & 1.24 & (3) & 5 \\
\hline 11. & 141.42079 & (20) & 7071.110 & (1) & 1.027 & (6) & 2 \\
\hline 12. & 140.67685 & (30) & 7108.49 & (2) & 0.680 & (6) & 2 \\
\hline 13. & 131.57953 & (40) & 7559.97 & (2) & 0.439 & (6) & 2 \\
\hline 14. & 126.8874 & (16) & 7881.0 & (1) & 0.55 & (1) & 5 \\
\hline 15. & 125.8099 & (31) & 7948.5 & (2) & 0.57 & (2) & 5 \\
\hline
\end{tabular}

Notes: (1) Possible rotational split mode.

(2) Fit using least squares program and effectively prewhitened.

(3) Fit using least squares program, but ambiguous prewhitening.

(4) Period lies on top of an alias but amplitude is significantly higher than alias.

(5) No fit with least squares program. 
Table 4. Frequency splittings.

\begin{tabular}{lllr}
\hline Splitting & $\delta \nu(\mu H z)$ & Alias & $\delta \nu(\mu H z)$ \\
\hline$f_{7}-f_{1}$ & 216.26 & $1 / 2 \Pi_{\text {orbit }}$ & 229.15 \\
$f_{6}-f_{2}$ & 113.4 & $1 / \Pi_{\text {orbit }}$ & 114.58 \\
$f_{5}-f_{3}$ & 11.09 & $1 /$ day & 11.57 \\
\hline
\end{tabular}

Note: Table 4 shows the splittings found in the data and comparable aliases. The first splitting $\left(f_{7}-\right.$ $f_{1}$ ) is likely a rotational split mode, while the other two are modes that are affected by windowing.

\section{DATA ANALYSIS}

Pulsating sdB stars, though intriguing, have been problematic. The period range in which modes are detected can cover radial and non-radial $p$ - and $g$-modes. Add to this the fact that some pulsating sdB stars have a pulsation spectrum too dense to be explained by radial and low-order non-radial modes alone. PG 1336 was chosen as a WET target with the hope that it would simplify some of these complications. It was hoped that we could then use the known rotation rate to look for rotationally split modes, thus deciphering the radial from non-radial modes of pulsation. As indicated in Table 3 , it appears that one such mode was detected. Unfortunately, this only makes us ask why we found one split mode, but no others. There are too many modes for the rest to be radial. To indicate the type of problems encountered, Table 5 lists pulsation periods detected in a model with properties similar to PG 1336.

Table 5. Model pulsation periods for $M=0.4705 M_{\odot}$,

$$
T_{\text {eff }}=32,600 K, \log g=5.70 \text {. }
$$

\begin{tabular}{cccc}
\hline$n$ & $l$ & Period(s) & Splitting coeff. \\
\hline 0 & 0 & 243 & \\
1 & 0 & 184 & \\
9 & 1 & 112.4 & 0.6 \\
6 & 1 & 144.5 & 0.8 \\
5 & 1 & 174.5 & 0.9 \\
9 & 2 & 119.6 & 7.7 \\
7 & 2 & 137.5 & 6.5 \\
7 & 2 & 171.6 & 2.3 \\
5 & 2 & 185.1 & 25 \\
\hline
\end{tabular}




\section{CONCLUSIONS}

XCOV 17 provided a wealth of data on PG 1336 which asked as many questions as it answered. Fifteen separate pulsation modes were detected, with one pair which is likely an $m= \pm 1$ rotationally split pair. However, we have to ask: if there are 15 modes, too many for purely radial modes, then why only one rotationally split pair? If we are most likely to see $m= \pm 1$ due to orientation, are we preferentially seeing only $m=+1$ or $m=-1$, and if so, why?

So what lies ahead for PG 1336? We have successfully resolved the vast majority of the pulsations, but will we be able to make it mean anything, or is this WET run that requires another WET run to produce results? At this point, there is no answer to this question. SDK and MDR are working on extracting pulsations from the eclipses. Since this is one of the very few stars for which we can resolve the surface (due to the eclipses), we hope that the eclipses will help in separating radial from non-radial modes. Unfortunately, models produced by SDK and MDR place doubt on the success of such a venture.

In order for pulsating sdB stars to provide constraints on models, pulsation spectra will have to provide mode identification. At this point, there has only been one case where a few modes have been identified, and it is the extreme member of the class. It sheds no light on the remaining members of the class. So how can we identify modes, and better yet, extend mode identification past individual members? By providing constraints on surface gravity and age.

At this point, the error bars on the surface gravity (due to inconsistent atmospheric models) are such that nine members of the class have overlapping error bars. Models indicate that surface gravity is the leading factor in determining where the fundamental radial mode lies, so until we can reduce the error bars of the surface gravity, we cannot discern between the vast majority of pulsators (see Heber et al. these proceedings).

The outlook for age determination is somewhat better. Model evolutionary tracks have degeneracies, but preliminary identification of pulsators in open clusters may help. 
ACKNOWLEDGMENTS. Among the many supporters of the observations reported here, we would like to acknowledge support from the U.S. National Science Foundation through grant NSFYI/AST 9257049 to Iowa State University, and support from UNESCO through the International Institute of Theoretical and Applied Physics at Iowa State. MDR would also like to thank Y. Oda and the McDonald Observatory TAC for a generous allocation of telescope time.

\section{REFERENCES}

Downes R. A. 1986, ApJS, 61, 569

Green R.F., Schmidt M., Liebert J. 1986, ApJS, 61, 305

Kilkenny D., O'Donoghue D., Koen C., Lynas-Gray A.E., van Wyk F. 1998, MNRAS, 296, 329

Nather R. E., Winget D. E., Clemens J. C., Hansen C. J., Hine B. P. 1990, ApJ, 361, 309

O'Donoghue D., Koen C., Kilkenny D., Stobie R. S. 1999 in 11th European Workshop on White Dwarfs, eds. J.-E. Solheim \& E. G. Meištas, ASP Conference Series, Vol. 169, p. 149 
